\title{
Análisis psicométrico de la versión española del Brief Sexual Function Inventory (BSFI) en una muestra de hombres con historia de abuso de drogas
}

\section{Psychometric analysis of the Spanish version of the Brief Sexual Function Inventory (BSFI) in a male drug abuse sample}

Pablo Vallejo-Medina ${ }^{1}$; Alejandro Guillén-RIQuelme'; Juan Carlos Sierra ${ }^{1}$
| ${ }^{1}$ Universidad de Granada.

Enviar correspondencia a:

Juan Carlos Sierra. Facultad de Psicología. Universidad de Granada.

18071 Granada (España). E-mail: jcsierra@ugr.es

\section{RESUMEN}

Ante la ausencia de estudios psicométricos de autoinformes que evalúen la respuesta sexual en población drogodependiente, se propone como objetivo llevar a cabo el primer análisis psicométrico del Brief Sexual Function Inventory (BSFI) en una muestra de 104 consumidores de drogas. Los participantes contestaron al BSFI junto con el Changes in Sexual Functioning Questionnaire (CSFO-14) y el Addictive Behavior Questionnaire (ABO). El BSFI presenta una estructura factorial similar a la propuesta por los autores originales formada por cinco factores (Impulso sexual, $\alpha=0,75$; Satisfacción, $\alpha=0,63$; Erección, $\alpha=0,70$; Eyaculación, $\alpha=0,74$; y Problemas, $\alpha=0,70)$, aunque hay dos items que cambian de factor con respecto a la propuesta original. En cuanto a las evidencias de la validez de sus medidas, sus puntuaciones correlacionaron de forma significativa con las del CSFQ-14. Se discute la capacidad del BSFI para diferenciar entre muestras clínicas y no clínicas.

\section{ABSTRACT}

It is well known that there is a lack of psychometric studies testing sexual function in drug-dependent population. Thus, the main objective of this work was to carry out the first psychometric analysis of the Brief Sexual Functioning Inventory (BSFI) in a sample of drug users ( $N$ $=104$ ). Participants filled out the BSFI, Changes in Sexual Functioning Questionnaire (CSFO-14) and Addictive Behavior Questionnaire (ABO). The BSFI shows a five-factor structure similar to the original (Sexual drive, $\alpha=.75$; Satisfaction, $\alpha=.63$; Erectile function, $\alpha=.70$; Ejaculatory function, $\alpha=.74$; and Problem Assessment, $\alpha=.70$ ), though compared to the original scale two items are loading in a different dimension. The high and significant correlations with the CSFO-14 indicated adequate validity of the measures. Capacity of the BSFI to discriminate between clinical and non-clinical samples is discussed.

Key words: BSFI, sexual functioning, drugs, instrumental studies. 


\section{INTRODUCCIÓN}

En la práctica clínica se viene observando durante años una relación directa entre el consumo de drogas y la salud sexual de los pacientes. Peugh y Belenko (2001) mostraron evidencias de que el consumo de sustancias afecta al comportamiento sexual. La influencia es tal que, en drogodependientes, el riesgo de padecer disfunción eréctil es 10 veces mayor que en la población normal (La Pera, Giannotti, Taggi y Macchia, 2003). En España, al existir una prevalencia de consumo elevada (primer pais consumidor mundial de cocaína y segundo europeo de cannabis, según el informe de Organización de Naciones Unidas (2007), los problemas sexuales producidos por el consumo de sustancias serían elevados. Asimismo, es común el consumo de estas sustancias con fines sexuales (Calafat, Juan, Becoña y Mantecon, 2008; Rawson, Washton, Domier y Reiber, 2002).

El efecto de las drogas en la sexualidad humana ha sido ampliamente investigado, evidenciándose una causalidad entre su consumo y la salud sexual. Por un lado, se ha observado que, a dosis bajas y a corto plazo, el alcohol (Emanuele y Emanuele, 1998), las benzodiacepinas (Smith, 2007), el cannabis (Lévy y Garnier, 2006; McKay, 2005), la cocaína (Buffum, Moser y Smith, 1988; Peugh y Belenko, 2001; Rosen, 1991), los opioides (Hyatt y Bensky, 1999), las anfetaminas y las metanfetaminas (Degenhardt y Topp, 2003; Kurtz, 2005), y los alucinógenos (Lévy y Garnier, 2006) pueden llegar a mejorar diversas áreas del funcionamiento sexual, aunque habitualmente estos efectos son efímeros ya que se produce una rápida habituación. A dosis más elevadas y/o en periodos de tiempo más prolongados, los efectos de las drogas son devastadores, afectando tanto a los aspectos físiológicos del ciclo sexual (Argiolas, 1999; Crenshaw y Goldberg, 1996; Cocores, Miller, Pottash y Gold, 1988; Johnson, Phelps y Cottler, 2004), como a los aspectos psicológicos de la sexualidad (Aguilar et al., 2008; Ávila Escribano, Pérez Madruga, Ólazabal Ulacia y López Fidalgo, 2004; La Pera et al., 2003; Lévy y Garnier, 2006; Peugh y Belenko, 2001; Rosen, 1991; Smith, 2007). Asimismo, es importante destacar la influencia que el tabaco tiene sobre la respuesta sexual humana, tanto a corto, como a largo plazo (Gades et al., 2005; Shiri et al., 2004).

La sexualidad es una dimensión compleja y multifacética del ser humano, donde interactúan variables psicológicas, biológicas, sociales e interpersonales (Basson, 2004), de forma que sólo bajo un estricto equilibrio, ésta acontecerá de forma natural y sana. De esta manera, cualquier agente que irrumpa en dicho equilibrio (estrés, problemas de pareja, rutina, alteraciones biológicas, etc.) provocará una alteración en la función sexual. Las drogas pueden afectar a cualquiera de las cuatro facetas propuestas por Basson; quizá ésta sea la explicación del gran efecto perturbador que su consumo ejerce sobre la sexualidad. Con el fin de recabar información sobre la sexualidad humana, recogiendo las particularidades de una población tan peculiar como la drogodependiente, en la presente investigación se ha considerado el planteamiento de Carrobles y Sanz (1991) como el modelo teórico de la respuesta sexual en donde se asentaría el Brief Sexual Function Inventory (BSFI; O'Leary et al., 1995), pues contempla tanto la dimensión fisiológica de la excitación y del orgasmo, como la psicológica del deseo y la satisfacción sexual.

EI BSFI (O'Leary et al., 1995) se elaboró a partir de más de 50 ítems, reducidos a 41 tras una primera revisión. Para analizar sus propiedades psicométricas, estos items se administraron a 144 sujetos. Después de este segundo análisis, el cuestionario se vio reducido a 22 ítems, los cuales finalmente, y tras una nueva revisión psicométrica en una muestra de 134 sujetos, dieron lugar a la versión definitiva de 11 items, los cuales se agruparon en cinco dimensiones: Impulso sexual ( 2 items, $\alpha=0,88$ ), Función eréctil ( 3 ítems, $\alpha=0,95$ ), Eyaculación (2 items, $\alpha=0,62$ ), Problemas (3 items, $\alpha=0,81$ ) y Satisfacción global (1 item). La fiabilidad test-retest, con una semana de intervalo, fluctuó entre 0,79 y 0,89 . El BSFI mostró además una adecuada validez discriminante por grupos de edad (O'Leary et al., 1995, 2003). Posteriormente, Mykletun, Dahl, O'Leary y Fossa (2005) plantean una estructura trifactorial (Impulso, Erección y Eyaculación), una vez descartados los ítems referidos a problemas y satisfacción; esta solución llega a explicar el $87 \%$ de la varianza. No obstante, estos mismos autores consideran más adecuada una estructura unidimensional -una vez excluido el ítem referido a satisfacción-, la cual explica un $66 \%$ de la varianza y presenta una fiabilidad de consistencia interna de 0,94.

Como se ha señalado, existe un gran volumen de investigación acerca de los efectos de las drogas sobre la sexualidad humana, por lo que resulta cuanto menos curioso que no exista ningún autoinforme de funcionamiento sexual validado en población drogodependiente, más aún tratándose de una población con unas características idiosincrásicas tan marcadas que la diferencian sustancialmente de la población normal. Uno de los métodos que mejor equilibrio presta entre precisión y viabilidad en la evaluación de la sexualidad humana son los autoinformes, los cuales constituyen una constante en la investigación psicológica. Por tanto, este estudio instrumental (Montero y León, 2007) pretende analizar algunas propiedades psicométricas del BSFI en una muestra española de consumidores de drogas. Para ello, y siguiendo los planteamientos de Carretero-Dios y Pérez (2007), se llevará a cabo un análisis de su fiabilidad y se examinará su validez de constructo; como muestra de validez convergente, se espera encontrar una relación significativa y elevada entre el BSFI y el Changes in Sexual Functioning Questionnaire (CSFQ-14; Keller, McGarvey y Clayton, 2006), el cual fue validado en población española por Bobes et al. (2000); finalmente, poniendo a prueba la validez discriminante, se espera encontrar puntuaciones más bajas que las halladas en muestras de población normal y similares a las de muestras clínicas de otros estudios (Mykletun et al., 2005; 0'Leary et al., 2003). 


\section{MÉTODO}

\section{Participantes}

La muestra se compone de 104 sujetos con historial de consumo de drogas, con una edad media de 31,91 años (DT= $9,14)$, siendo la edad mínima 17 y la máxima 63 . Con el objetivo de evaluar a una muestra heterogénea de consumidores, se incluyeron tanto sujetos en abstinencia, como aquellos que abusaban actualmente, así como sujetos en tratamiento residencial, ambulatorio o libres de éste. El reclutamiento de los sujetos en tratamiento residencial y ambulatorio se realizó, mediante muestreo por conglomerados, en el recurso de la Junta de Andalucía Cortijo Buenos Aires de Granada, en Proyecto Hombre Granada, y en la Unidad de Trastornos Emocionales y Adictivos del Hospital Internacional Medimar de Alicante; los sujetos sin tratamiento fueron reclutados mediante muestreo incidental. El criterio de inclusión para que un sujeto fuera considerado consumidor era que superase los siguientes umbrales de consumo mínimo (descritos según las distintas sustancias de abuso):

- Alcohol. Según los criterios del Observatorio Español sobre Drogas (OED) (2007) el límite sería un consumo mínimo de 40 gramos de alcohol al día (aproximadamente 1 litro de cerveza).

- Cannabis. Según el Observatorio Andaluz sobre Drogas y Adicciones (OADA) (2007), el consumo mínimo para considerar uso habitual sería consumir la sustancia más de un día a la semana.
- Anfetaminas y derivados. Según el informe del Observatorio Andaluz sobre Drogas y Adicciones (OADA) (2007) el consumo de estas drogas responde a un patrón de consumo experimental, contabilizándose muy pocos casos de consumo diario e incluso semanal. Por ello, el criterio de inclusión fue un consumo superior a una vez al mes.

- Alucinógenos. Según Ballesta, Lozano, Bilbao y González (2004) el consumo de alucinógenos es principalmente experimental, por lo que el criterio de inclusión será el mismo que para la anfetaminas (más de una vez al mes).

- Cocaína, opioides y combinaciones. En este caso, el criterio fue arbitrario, situándolo en uno o más consumos semanales.

Además, para su inclusión en el estudio, los participantes debían encontrarse libres del efecto agudo de la sustancia, es decir, no haber consumido drogas durante las 24 horas previas a la evaluación (se permitía 1,5 gramos de alcohol). El control de esta variable se hizo a través de análisis de muestras de orina en los sujetos bajo tratamiento y mediante autoinforme verbal en el resto de participantes. En cuanto al consumo de tabaco, a pesar de que no se ha utilizado como criterio de inclusión, sí se ha contabilizado su tasa de igual modo que en el resto de sustancias. Las tasas de consumo y los datos sociodemográficos de la muestra aparecen en la Tabla 1.

Tabla 1. Datos sociodemográficos de la muestra.

\begin{tabular}{|c|c|c|c|c|c|c|c|c|c|c|c|}
\hline Características & Cannabis & Benzodiacepinas & Cocaína & Anfetaminas & Heroína & Metadona & Alucinógenos & Combinaciones & Tabaco & Alcohol & Total \\
\hline Sujetos & 87 & 30 & 80 & 37 & 31 & 21 & 51 & 28 & 91 & 90 & 104 \\
\hline Severidad (DT) & $7,13(9,86)$ & $12,45(19,73)$ & $5,59(5,59)$ & $2,54(4,33)$ & $4,57(3,9)$ & $120,8(127,51)$ & $0,51(1,05)$ & $5,54(4,32)$ & $18,22(8,34)$ & $39,55(53,79)$ & (...) \\
\hline Minimo & 0,01 & 0,03 & 0,03 & 0,01 & 0,01 & 0,01 & 0,1 & 0,1 & 1 & 0,08 & $-3,2$ \\
\hline Máximo & 57,21 & 88,27 & 19,62 & 22,6 & 14,71 & 392,4 & 5,04 & 16,35 & 40 & 304,04 & 9,81 \\
\hline Sustancia preferente & 19 & 1 & 36 & 0 & 7 & 1 & 0 & 10 & 3 & 24 & \\
\hline Edad (DT) & $30,97(8,49)$ & $36,33(7,39)$ & $31,91(7,91)$ & $32,26(8,87)$ & $36,45(7,23)$ & $36,19(6,92)$ & $31,69(8,08)$ & $37,54(6,57)$ & $32,46(9,14)$ & $32,23(9,32)$ & $31,9(9,19)$ \\
\hline \multicolumn{12}{|l|}{ Estado civil } \\
\hline Casado & 9 & 4 & 10 & 3 & 4 & 1 & 4 & 4 & 12 & 13 & 14 \\
\hline Soltero & 63 & 17 & 55 & 25 & 21 & 15 & 40 & 18 & 16 & 63 & 73 \\
\hline Divorciado & 14 & 9 & 15 & 9 & 6 & 5 & 7 & 6 & 62 & 13 & 16 \\
\hline \multicolumn{12}{|l|}{ Nivel educativo } \\
\hline Primaria & 32 & 17 & 34 & 16 & 18 & 15 & 19 & 18 & 36 & 37 & 40 \\
\hline Secundaria & 19 & 5 & 18 & 10 & 8 & 4 & 14 & 7 & 18 & 19 & 21 \\
\hline CFGS & 8 & 4 & 9 & 5 & 1 & 1 & 5 & 1 & 10 & 5 & 10 \\
\hline Universitario & 25 & 4 & 15 & 6 & 2 & 1 & 12 & 1 & 21 & 23 & 27 \\
\hline Con medicación & 35 & 14 & 36 & 20 & 16 & 10 & 26 & 14 & 31 & 37 & 44 \\
\hline $\begin{array}{l}\text { Años medios } \\
\text { consumo (DT) }\end{array}$ & $12,4(8,22)$ & $11,1(8,91)$ & $11,1(8,06)$ & $8,1(7,28)$ & $11,7(8,35)$ & $5,6(6,86)$ & $4,8(6,41)$ & $12,6(8,34)$ & $16,91(9,41)$ & $15,6(9,39)$ & $10,32(7,98)$ \\
\hline Máximo tiempo & 31,5 & 28 & 29,42 & 29,42 & 24 & 25 & 29,42 & 26,67 & 44 & 38 & 44 \\
\hline \multicolumn{12}{|l|}{ Via administración } \\
\hline Oral & 2 & 30 & 5 & 36 & 1 & 21 & 51 & 0 & 2 & 53 & $g^{c}$ \\
\hline Esnifada & 0 & 1 & 74 & 12 & 9 & 0 & 0 & 5 & & 0 & $5^{c}$ \\
\hline Inyectada & 0 & 1 & 7 & 0 & 8 & 0 & 0 & 4 & & 1 & $5^{c}$ \\
\hline Fumada & 87 & 1 & 34 & 0 & 28 & 0 & 0 & 26 & 86 & 0 & $6^{c}$ \\
\hline Inhalada & 0 & 0 & 6 & 0 & 5 & 0 & 1 & 4 & & 1 & $5^{c}$ \\
\hline Consumo último mes & $24,1 \%$ & $30 \%$ & $12 \%$ & $7,9 \%$ & $6,7 \%$ & $10 \%$ & $7,8 \%$ & $3,6 \%$ & $91 \%$ & $37 \%$ & (...) \\
\hline
\end{tabular}

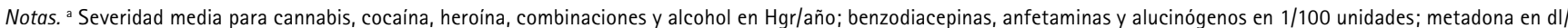
año; tabaco en cigarrillos/dia. ${ }^{b}$ Sin contabilizar los periodos de abstinencia. ${ }^{c}$ Total de sustancias que se han administrado mediante una determinada vía. DT: desviación típica, CFGS: Ciclo Formativo de Grado Superior. 


\section{Instrumentos}

- Brief Sexual Function Inventory (BSFI; O'Leary et al., 1995). Sus 11 items, contestados en una escala tipo Likert de 5 alternativas de respuesta variable, evalúan la respuesta sexual masculina. Proporciona puntuaciones en cinco dimensiones teóricas de la sexualidad humana: Impulso sexual (items 1 y 2), Erección (items 3,4 y 5), Eyaculación (ítems 6 y 7), Problemas sexuales (items 8,9 y 10) y Satisfacción (item 11). Puntuaciones elevadas indican un mejor funcionamiento sexual. Véase Anexo 1.

- Addictive Behavior Questionnaire (ABO; López-Torrecillas, Godoy, Pérez-García y Godoy, 2000). Permite recoger el tipo de sustancia que el sujeto consume, la vía de administración, la severidad y la cronicidad del consumo. En el presente estudio se incluyó también un periodo de abstinencia y se realizaron algunos cambios sobre el listado de sustancias incluidas, eliminando la medicación y añadiendo combinaciones, benzodiacepinas y tabaco, por lo que finalmente contempla como posibles sustancias de consumo el cannabis, las benzodiacepinas, la cocaina, las anfetaminas, la heroína, la metadona, los alucinógenos, las combinaciones, el tabaco y el alcohol. El formato de respuesta incluye tanto opciones preestablecidas con posible respuesta múltiple, como preguntas de respuesta abierta.

- Changes in Sexual Functioning Questionnaire (CSFO14; Keller et al., 2006). Este cuestionario está constituido por 14 items contestados en una escala de tipo Likert con 5 alternativas de respuesta variable, que permiten evaluar el funcionamiento sexual. Sus autores agrupan de forma teórica los items en cinco dimensiones (Placer, item 1; Deseo-frecuencia, items 2 y 3; Deseo-interés, ítems 4, 5 y 6; Excitación, items 7, 8 y 9); y Orgasmo, items 11, 12 y 13) o en tres fases del ciclo sexual (Deseo, ítems 1 a 5; Excitación, items 7 a 9 ; y Orgasmo, items 11 a 13). El cuestionario presenta una forma masculina y otra femenina; en este caso, y dadas las caracteristicas de la muestra, se ha empleado la masculina. En la validación de la versión española extendida del CSFQ, Bobes et al. (2000) informan de las cinco dimensiones descritas, oscilando sus coeficientes de consistencia interna entre 0,75 y 0,82 , dependiendo de la subescala. Recientemente, Vallejo-Medina, Guillén-Riquelme y Sierra (2009), mediante análisis factorial exploratorio, aíslan únicamente tres subescalas que explican el $56,37 \%$ de la varianza: Deseo $(\alpha=0,73)$, Excitación-orgasmo ( $\alpha=$ $0,67)$ y Placer (compuesta por un solo item).

\section{Procedimiento}

En primer lugar, se procedió a la adaptación lingüistica del BSFI empleando para ello la traducción hacia delante (Hambleton, 1996). Primero se tradujeron los items al casteIlano, dicha traducción se entregó, junto al original en inglés, a un experto bilingüe que sugirió una serie de cambios. Se modificó el original con los cambios sugeridos, culminando de este modo el proceso de traducción. A continuación se envió el cuestionario a cuatro jueces expertos en sexualidad, quienes juzgaron la adecuación y comprensión de los items proponiendo, si fuese necesaria, una redacción alternativa. El siguiente paso consistió en calcular el porcentaje de acuerdo, revisándose según las recomendaciones de los expertos aquellos ítems que no alcanzaron el 85\% de acuerdo. La batería final se entregó a 10 hombres adultos quienes juzgaron nuevamente la comprensión de los ítems, señalando aquellos que no entendian; nuevamente se revisaron los ítems que contaban con un consenso inferior al 85\%.

Tras finalizar la traducción y adaptación, los tres cuestionarios integraron un cuadernillo junto al consentimiento informado (que garantizaba el anonimato). La administración fue realizada de forma individual por un único entrevistador. Las instrucciones, tanto orales como escritas, fueron similares para cada uno de los sujetos. La duración de la aplicación completa fue de 20 minutos. Una vez cumplimentado el cuadernillo se plegaba sobre sí mismo y se introducía en una urna sellada para garantizar el anonimato y promover la sinceridad. Un total de 12 sujetos rehusaron participar en el estudio.

\section{RESULTADOS}

Como se aprecia en la Tabla 2, las puntuaciones medias de los ítems se sitúan en torno a la media teórica de dos, sobrepasando únicamente cinco ítems $(5,6,7,9$ y 10$)$ el valor de tres. Las desviaciones típicas se aproximan a uno, superando dicho valor seis de los once ítems, tal como es aconsejable (Carretero-Dios y Pérez, 2005).

Tabla 2. Propiedades psicométricas de los ítems del BSFI ( $M$ : media; $D T$ : desviación tipica; $I D$ : índice de discriminación; $\alpha$ : alfa de Cronbach si se elimina el ítem).

\begin{tabular}{lcccc}
\hline Items & $\mathrm{M}$ & $\mathrm{DT}$ & $\mathrm{ID}$ & $\alpha$ \\
\hline BSFI-1 & 2,83 & 1,05 & 0,38 & 0,83 \\
BSFI-2 & 2,84 & 0,99 & 0,48 & 0,82 \\
BSFI-3 & 2,47 & 1,19 & 0,58 & 0,81 \\
BSFI-4 & 2,74 & 1,25 & 0,69 & 0,80 \\
BSFI-5 & 3,41 & 0,88 & 0,54 & 0,82 \\
BSFI-6 & 3,23 & 0,96 & 0,51 & 0,82 \\
BSFI-7 & 3,35 & 0,94 & 0,54 & 0,82 \\
BSFI-8 & 2,20 & 1,50 & 0,45 & 0,83 \\
BSFI-9 & 3,31 & 0,95 & 0,54 & 0,82 \\
BSFI-10 & 3,24 & 1,08 & 0,38 & 0,83 \\
BSFI-11 & 2,36 & 1,28 & 0,63 & 0,81 \\
\hline
\end{tabular}

En cuanto a la fiabilidad de consistencia interna, se obtiene un alfa de Cronbach de 0,83 , no existiendo ningún ítem que mejore dicho valor si se elimina. Este valor se considera adecuado según Nunnally y Bernstein (1995), quienes postu- 
lan que un coeficiente entorno a 0,70 sería adecuado para la investigación, mientras que si el fin es clínico, el coeficiente debería rondar el valor de 0,80 . Los índices de discriminación superan en todos los casos el valor de 0,30.

Para comprobar los supuestos pertinentes a la reducción factorial se ha utilizado la prueba de Kaiser-Meyer-Olkin (KMO $=0,77)$, así como la prueba de esfericidad de Bartlett $\left(\chi^{2}{ }_{55}\right.$ $=427,56 ; p<0,001)$, que mostraron que los datos son adecuados para la realización de un análisis factorial. Además, mediante las correlaciones entre ítems, se comprobó que no existen problemas de multicolinealidad. El procedimiento seguido fue el método de componentes principales, con rotación Varimax, forzando a cinco factores, que es la estructura factorial defendida originalmente por O'Leary et al. (1995). De los cinco factores aislados únicamente tres superan un valor propio superior a uno, pese a ello se mantendrá dicha factorización en concordancia a la factorización original. Estos cinco factores explican un $79,61 \%$ de la varianza total. Las saturaciones de la reducción factorial se muestran en la Tabla 3.

Tabla 3. Matriz de componentes rotados forzado a $\mathbf{5}$ factores.

\begin{tabular}{lcccccc}
\hline Items & $\begin{array}{r}\text { Impulso } \\
\text { sexual }\end{array}$ & Satisfacción & Erección & Eyaculación & Problemas & Comunalidades \\
\hline BSFI-1 & 0,88 & & & & 0,79 \\
BSFI-2 & 0,87 & & & & 0,78 \\
BSFI-3 & 0,69 & 0,30 & & & 0,65 \\
BSFI-8 & & 0,86 & & & 0,69 \\
BSFI-11 & & 0,83 & & & 0,85 \\
BSFI-5 & & & 0,87 & & 0,83 \\
BSFI-4 & 0,39 & 0,32 & 0,58 & 0,30 & & 0,81 \\
BSFI-6 & & & & 0,84 & & 0,81 \\
BSFI-7 & & & & 0,80 & 0,33 & 0,81 \\
BSFl-10 & & & & & 0,89 & 0,85 \\
BSFI-9 & & & 0,61 & & 0,63 & 0,83 \\
\hline $\begin{array}{l}\text { \% varianza } \\
\text { explicada }\end{array}$ & 39,38 & 15,70 & 10,19 & 8,14 & 6,17 & \\
$\begin{array}{l}\text { Valor } \\
\text { propio }\end{array}$ & 4,33 & 1,72 & 1,12 & 0,89 & 0,67 & \\
$\begin{array}{l}\text { Alfa } \\
\text { Cronbach }\end{array}$ & 0,75 & 0,63 & 0,70 & 0,74 & 0,70 & \\
\hline
\end{tabular}

Notas. Para mayor claridad de la tabla se han eliminado las saturaciones inferiores a 0,30 . En negrita aparecen las cargas factoriales en el factor correspondiente.

Podemos observar que la distribución de las cargas factoriales de los items mantiene la estructura hipotetizada por 0'Leary et al. (1995), salvo el ítem 3 que semánticamente debería posicionarse en el factor Erección, pero carga en la dimensión Impulso sexualy el ítem 8 que en el presente estudio carga en el factor Satisfacción, cuando semánticamente encajaría en el componente Problemas. Así pues, a excepción de estos dos ítems, la factorización original parece mantenerse.

Como se observa en la Tabla 4, todos los ítems alcanzan un índice de discriminación superior a 0,50, reafirmando el poder discriminante de los 5 factores. Se puede apreciar también como todos los ítems refuerzan la consistencia interna de la dimensión a la que pertenecen, salvo el ítem
3. cuya eliminación mejoraría el alfa de Cronbach de la dimensión Impulso sexual, pasando de 0,75 a 0,82. Debemos recordar que el ítem 3 carga en un factor erróneo, por lo que probablemente hubiera que revisarlo. Ningún factor mejoraría la consistencia interna si fuese eliminado. Respecto a las correlaciones entre cada uno de los factores con el total del test, eliminando su puntuación del total, éstas se sitúan por encima de 0,30, siendo la menor de 0,38, por lo que resultan adecuadas.

Tabla 4. Propiedades psicométricas de los factores del BSFI (ID: indice de discriminación; $\alpha$ : alfa de Cronbach si se elimina el ítem; M: media; DT: desviación típica).

\begin{tabular}{lcccc}
\hline Factores & Items & $I D$ & $\alpha$ & $M(D T)$ \\
\hline \multirow{4}{*}{ Impulso sexual } & BSFI-1 & 0,66 & 0,69 & \\
& BSFI-2 & 0,72 & 0,64 & \\
& BSFI-3 & 0,55 & 0,82 & \\
Satisfacción & Total & 0,38 & 0,75 & $8,13(2,71)$ \\
\hline \multirow{4}{*}{ Erección } & BSFI-8 & 0,60 & & \\
& BSFI-11 & 0,60 & & \\
& Total & 0,64 & 0,63 & $4,56(2,49)$ \\
\hline \multirow{3}{*}{ Eyaculación } & BSFI-4 & 0,52 & & \\
& BSFI-5 & 0,52 & & $6,14(1,87)$ \\
\hline & Total & 0,71 & 0,70 & \\
\hline \multirow{3}{*}{ Problemas } & BSFI-6 & 0,54 & & $6,40(1,89)$ \\
\hline & BSFI-7 & 0,54 & & $6,55(1,82)$ \\
\hline
\end{tabular}

Nota. No se puede calcular el alfa Cronbach si se elimina el item en factores con menos de 3 items.

Tabla 5. Correlaciones de los factores del BSFI entre sí y con la escala total.

\begin{tabular}{|c|c|c|c|c|c|}
\hline & $\begin{array}{c}\text { Impulso } \\
\text { sexual }\end{array}$ & Erección & $\begin{array}{l}\text { Eyacula- } \\
\text { ción }\end{array}$ & Problemas & Total BSF \\
\hline $\begin{array}{l}\text { Impulso } \\
\text { sexual }\end{array}$ & & & & & $0,57^{* *}$ \\
\hline Erección & $0,55^{* *}$ & & & & $0,86^{* *}$ \\
\hline $\begin{array}{l}\text { Eyacula- } \\
\text { ción }\end{array}$ & $0,28^{* *}$ & $0,50^{* *}$ & & & $0,71^{* *}$ \\
\hline Problemas & 0,08 & $0,44^{* *}$ & $0,43^{* *}$ & & $0,72^{* *}$ \\
\hline $\begin{array}{l}\text { Satisfac- } \\
\text { ción }\end{array}$ & $0,25^{* *}$ & $0,55^{* *}$ & $0,44^{* *}$ & $0,55^{* *}$ & $0,73^{* *}$ \\
\hline
\end{tabular}

${ }^{* *} p<0,01$.

Se han calculado también las correlaciones entre los factores, así como entre cada uno de ellos y el total (véase la Tabla 5). Las correlaciones entre factores se sitúan en torno a 0,50 o menos, por lo que se puede inferir cierta relación entre las dimensiones, aunque no con la suficiente magnitud que haga dudar de la multidimensionalidad de la escala. 
Por último, se calcularon la media y la desviación típica para la escala total del BSFI $(M=31,84 ; D T=7,61)$ y para cada una de las diferentes dimensiones: Impulso sexual $(M=$ 8,13; $D T=2,71)$, Satisfacción $(M=4,56 ; D T=2,49)$, Erección $(M=6,14 ; D T=1,87)$, Eyaculación $(M=6,40 ; D T=1,89)$ y Problemas $(M=6,55 ; D T=1,82)$.

\section{Indicios de validez del BSFI}

Con el objetivo de examinar la validez convergente, se correlacionaron las puntuaciones del BSFI con las del CSFO14, resultando todas ellas significativas (véase la Figura 1), oscilando entre 0,37 y 0,64 .

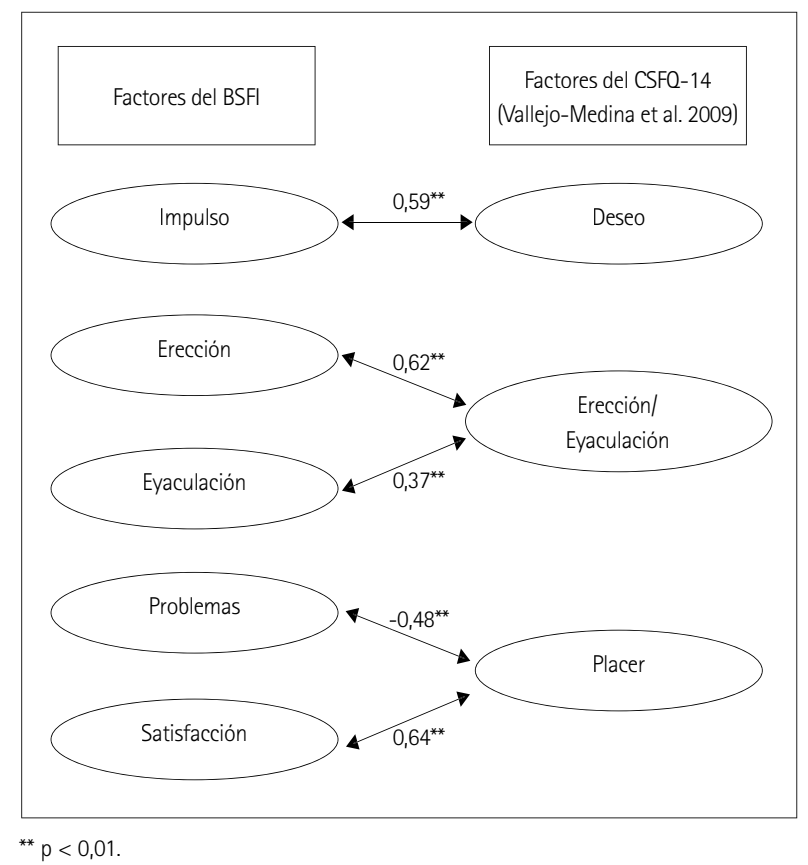

Figura 1. Correlaciones entre los factores del CSFO-14 y el BSFI.

\section{DISCUSIÓN}

El objetivo de este estudio ha sido adaptar y aportar algunas evidencias sobre las propiedades métricas del Brief Sexual Function Inventory, BSFI (O'Leary et al., 1995) en una muestra de hombres con historia de abuso de drogas, con el fin de obtener, de este modo, las primeras propiedades psicométricas de un autoinforme de funcionamiento sexual en hombres consumidores de drogas. Dicho objetivo ha sido cubierto mediante la adaptación y evaluación cualitativa de los ítems, el análisis de las propiedades psicométricas de los mismos, la estimación de la fiabilidad y la validez de constructo, así como de algunos indicadores de validez externa.

Todos los ítems del BSFI presentaron un índice de discriminación adecuado, siendo además indudable su aportación individual a la consistencia interna, ya que la supresión de algún ítem va a repercutir en el alfa de Cronbach global $(0,83)$, disminuyéndolo. Este valor es adecuado, aunque resulta inferior al informado por Mykletun et al. (2005), quienes obtienen un coeficiente de 0,94 una vez eliminado el ítem 11.

El análisis factorial forzado a cinco factores, como O'Leary et al. (1995) hipotetizaban, arrojó una estructura concordante a excepción de los ítems 8 (En los últimos 30 días, ¿en qué medida ha considerado como problemática la falta de actividad sexual?) y 3 (Durante los últimos 30 días, ¿con qué frecuencia tuvo erecciones completas o parciales tras recibir cualquier forma de estimulación sexual?). Este último, pese a que la reducción factorial lo incluye en el factor Impulso sexual, podría pensarse que realmente no pertenece a ese factor, pues es el único ítem que si es eliminado mejoraria la consistencia interna del propio factor de forma significativa. Por tanto, se cuenta con un factor predominantemente de impulso sexual, otro de satisfacción sexual, con una fuerte interferencia del ítem 8, y tres factores coherentes para erección, eyaculación y problemas sexuales, oscilando sus coeficientes de fiabilidad entre 0,63 (Satisfacción) y 0,75 (Impulso sexual), valores inferiores a los indicados por 0'Leary et al. (1995). Mykletun et al. (2005) realizaron un análisis factorial, excluyendo los ítems referentes a problemas sexuales (ítems 8,9 y 19) y el ítem 11 (satisfacción general) obteniendo un modelo de tres factores claramente definidos (Impulso sexual, Erección y Eyaculación), aunque defienden un uso unidimensional de la escala, en base al elevado valor del alfa de Cronbach de la escala total. En nuestro estudio, el hecho de forzar cinco factores y considerar dos de ellos con valores propios inferiores a uno, hace que la robustez del modelo pentafactorial no quede claramente consolidada. En nuestro caso, y en la línea de lo defendido por Myckletun et al., también se ha probado la unidimensionalidad de la escala, pero se obtuvieron resultados poco concluyentes. Las correlaciones entre las dimensiones encontradas son ligeramente más bajas que las halladas por 0'Leary et al. (2003) por lo que se confirma la relación existente entre los factores, pese a que no son lo suficientemente elevadas como para proclamar la dependencia de éstas.

A la hora de comparar las puntuaciones del presente estudio con las obtenidas en otras investigaciones, se ha observado que la media total $(31,84 ; D T=7,61)$ es menor a la encontrada por 0'Leary et al. (2003) en población normal con edades entre 40-49 años $(M=35,5)$. Por su parte, en el estudio de Mykletun et al. (2005) se obtiene una media para los 10 ítems, en población noruega normal, de 3,50 (DT $=0,42$ ) en un grupo de edad de 30-39 años; mientras, en el presente estudio, la media para esos diez ítems fue 2,96 $(D T=1,08)$. En definitiva, se observa que los varones consumidores de droga puntúan más bajo en funcionamiento sexual que la población normal, tanto noruega como estadounidense. Estos datos parecen estar indicando una adecuada capacidad discriminante del cuestionario entre diversos tipos de muestras, aunque habría que ser cautos en esta interpretación, pues al no existir validaciones en población española resulta difícil comprobar la equivalencia entre las muestras de los distintos países. Finalmente, los resultados muestran evidencias de la validez convergente del instrumento, al encontrarse correlaciones significativas entre las puntuaciones del BSFI y del CSFO-14. 


\section{Limitaciones}

Pese a que los resultados parecen indicar una buena bondad psicométrica del BSFI en esta muestra de hombres consumidores de drogas, es necesario reseñar que los datos encontrados son exploratorios, marcando la tendencia de las puntuaciones, que difícilmente podrian enmarcarse como conclusiones definitivas. El tamaño muestral es relativamente bajo y heterogéneo con respecto a su edad, siendo necesario en trabajos futuros aumentar la muestra. De esta forma, se podrá ratificar la estructura factorial mediante análisis factorial confirmatorio, ampliar los datos sobre la fiabilidad y obtener baremos para población drogodependiente, en función de la edad y de la sustancia preferente de consumo. Asimismo, se debe incidir en el problema del policonsumo propio de la muestra evaluada, en su amplio rango de edad, así como en el registro aproximativo y autoinformado de la historia de consumo personal. Todas estas cuestiones deberian tenerse en consideración para futuros estudios.

\section{AGRADECIMIENTOS}

Los autores muestran su agradecimiento al Recurso de la Red de Servicios Sociales de la Junta de Andalucia "Cortijo Buenos Aires" de Granada, en especial a su director Francisco Aguilar de Arcos, a Proyecto Hombre Granada, en concreto a José María Tortosa Alarcón, su director, así como a Teresa Pereiro, María del Carmen Solivares y Sandra Owono, directoras de la Unidad de Trastornos Emocionales y Adictivos del Hospital Internacional Medimar de Alicante, por permitir y facilitar el acceso a la muestra.

\section{REFERENCIAS}

Aguilar, F., Verdejo-García, A., López Jiménez, A., Montañez Pareja, M., Gómez Juárez, E., Arráez Sánchez, F. y Pérez-García, M. (2008). Cambios en la respuesta emocional ante estímulos visuales de contenido sexual en adictos a las drogas. Adicciones, 20, 117124.

Argiolas, A. (1999). Neuropeptides and sexual behaviour. Neuroscience Et Biobehavioral Reviews, 23, 1127-1142.

Ávila Escribano, J.J., Pérez Madruga, A., Ólazabal Ulacia, J. C. y López Fidalgo, J. (2004). Disfunciones sexuales en el alcoholismo. Adicciones, 16, 1-6.

Ballesta, R., Lozano, Ó., Bilbao, I. y González, F. (2004). Estudio de evolución del informe de Los Andaluces ante las Drogas (19872003). Sevilla: Consejería para la Igualdad y Bienestar Social de la Junta de Andalucía.

Basson, R. (2004). Recent advances in women's sexual function and dysfunction. Menopause, 11, 714-725.

Bobes, J., González, M.P., Rico-Villademoros, F., Bascarán, M.T., Sarasa, P. y Clayton, A. (2000). Validation of the Spanish version of the Changes in Sexual Functioning Questionnaire (CSFO). Journal of Sex \& Marital Therapy, 26, 119-131.
Buffum, J., Moser, C. y Smith, D. (1988). Street drugs and sexual function. En J. Money, H. Musaph y J.M.A. Sitsen (Eds.), Handbook of Sexology (Vol.6). The pharmacology and endocrinology of sexual function (pp. 462-477). Nueva York: Elsevier Science Publishers.

Calafat, A., Juan, M., Becoña, E. y Mantecón, A. (2008). Qué drogas se prefieren para las relaciones sexuales en contextos recreativos. Adicciones, 20, 37-47.

Carretero-Dios, H. y Pérez, C. (2005). Normas para el desarrollo y revisión de estudios instrumentales. International Journal of Clinical and Health Psychology, 5, 521-551.

Carretero-Dios, H. y Pérez, C. (2007). Standards for the development and review of instrumental studies: Considerations about test selection in psychological research. International Journal of Clinical and Health Psychology, 7, 863-882.

Carrobles, J.A. y Sanz, A. (1991). Terapia sexual. Madrid: Fundación Universidad-Empresa.

Cocores, J.A., Miller, N.S., Pottash, A.C. y Gold, M.S. (1988). Sexual dysfunction in abusers of cocaine and alcohol. American Journal of Drug and Alcohol Abuse, 14, 169-173.

Crenshaw, T.L. y Goldberg, J.P. (1996). Sexual pharmacology: Drugs that affect sexual functioning. Nueva York, NY: W.W. Norton \& Company.

Degenhardt, L. y Topp, L. (2003). Cristal meth use among polydrug users in Sydney's danse partysubculture: Characteristics, use patterns and associated harms. International Journal of Drug Policy, 14, 17-24.

Emanuele, M.A. y Emanuele, N.V. (1998). Alcohol's effects on male reproduction. Alcohol Health Research World, 22, 195-201.

Gades, N.M., Nehra, A., Jacobson, D.J., McGree, M.E., Girman, C.J., Rhodes, T., Roberts, R.O., Lieber, M.M. y Jacobsen, S.J. (2005). Association between smoking and erectile dysfunction: $\mathrm{A}$ Population-based Study. American Journal of Epidemiology, 161, 346-351.

Hambleton, R.K. (1996). Adaptación de tests para su uso en diferentes idiomas y culturas: fuentes de error, posibles soluciones y directrices prácticas. En J. Muñiz, (Ed.), Psicometría (pp. 203238). Madrid: Universitas.

Hyatt, B. y Bensky, K.P. (1999). Illicit drugs and anesthesia. The Clinical Forum for Nurse Anesthetists, 10, 15-23.

Johnson, S.D., Phelps, D.L. y Cottler, L.B. (2004). The association of sexual dysfunction and substance use among a community epidemiological sample. Archives of Sexual Behavior, 33, 55-63.

Keller, A., McGarvey, E.L. y Clayton, A.H. (2006). Reliability and construct validity of the Changes in Sexual Functioning Questionnaire Short-Form (CSFO-14). Journal of Sex \& Marital Therapy, 32, 43-52.

Kurtz, S.P. (2005). Post-circuit blues: Motivations and consequemces of crystal meth use among gay men in Miami. AIDS and Behavior, 9, 63-72.

La Pera, G., Giannotti, C.F., Taggi, F. y Macchia, T. (2003). Prevalence of sexual disorders in those young males who later become drug abusers. Journal of Sex \& Marital Therapy, 29, 149-156.

Lévy, J.J. y Garnier, C. (2006). Drogues, médicaments et sexualité. Drogues, Santé et Société, 5, 11-48.

López-Torrecillas, F., Godoy, J.F., Pérez-Garcia, M., Godoy, D. y Sánchez-Barrera, M.B. (2000). Variables modulating stress and coping that discriminate drug consumers from low or nondrug consumers. Addictive Behaviors, 25, 161-165. 
McKay, A. (2005). Sexuality and substance use: The impact of tobacco, alcohol, and selected recreational drugs on sexual function. Canadian Journal of Human Sexuality, 14, 47-56.

Montero, I. y León, G. (2007). A guide for naming research studies in Psychology. International Journal of Clinical and Health Psychology, 7, 847-862.

Mykletun, A., Dahl, A.A., O'Leary, M.P. y Fossa, S.D. (2005). Assessment of male sexual function by the Brief Sexual Function Inventory. BJU International, 97, 316-323.

Nunnally, J.C. y Bernstein, I.J. (1995). Teoría psicométrica. Madrid: McGraw-Hill.

Observatorio Andaluz sobre Drogas y Adicciones (OADA) (2007). La población andaluza ante las drogas X. Sevilla: Junta Andalucia.

Observatorio Español sobre Drogas (OED) (2007). Situación y tendencias de los problemas de drogas en España. Madrid: Ministerio de Sanidad y Consumo.

O'Leary, M.P., Fowler, F.J., Lenderking, W.R., Barber, B., Sagnier, P.P., Guess, H.A. y Barry, M.J. (1995). A brief male sexual function inventory for urology. Urology, 46, 697-706.

O'Leary, M.P., Rhodes, T., Girman, C.J., Jacobson, D.J., Roberts, R.O., Lieber, M.M. y Jacobsen, S.J. (2003). Distribution of the Brief Male Sexual Inventory in community men. International Journal of Impotence Research, 15, 185-191.

Organización de Naciones Unidas (2007). World Drug Report. Office on Drugs and Crime. Ginebra: ONU.

Peugh, J. y Belenko, S. (2001). Alcohol, drugs and sexual function: A review. Journal of Psychoactive Drugs, 33, 223-232.

Rawson, R.A., Washton, A., Domier, C.0. y Reiber, C. (2002). Drug and sexual effects: Role of drug type and gender. Journal of Substance Abuse Treatment, 22, 103-108.

Rosen, R.C. (1991). Alcohol and drug effects on sexual response: Human experimental and clinical studies. Annual Review of Sex Research, 2, 119-179.

Shiri, R., Koskimäki, J., Hakama, M., Häkkinen, J., Huhtala, H., Tammela, T.L.J. y Auvinen, A. (2004). Effect of life-style factors on incidence of erectile dysfunction. International Journal of Impotence Research, 16, 389-394.

Smith, S. (2007). Drugs that cause sexual dysfunction. Psychiatry, 6, $111-114$.

Vallejo-Medina, P., Guillén-Riquelme, A. y Sierra, J.C. (2009). Psychometric properties of the Spanish version of Changes in Sexual Functioning Questionnaire short-form (CSFO-14) among male drug abuse sample. Manuscrito sometido a publicación.

\section{Anexo 1. Versión española del BSFI.}

1. Durante los últimos 30 días, ¿Cuántos días ha experimentado impulsos sexuales? 0. Ningún dia 1 . Pocos dias 2. Algunos dias 3. La mayoría de los dias 4. Casi todos los días

2. Durante los últimos 30 días, ¿Cómo valoraría su nivel de impulso sexual? 0. Nulo 1. Bajo 2. Medio 3. Medio-Alto 4. Alto

3. Durante los últimos 30 días, ¿Con qué frecuencia tuvo erecciones completas o parciales tras recibir cualquier forma de estimulación sexual?

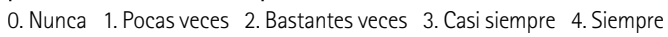

4. Durante los últimos 30 dias, cuando tuvo erecciones, ¿Con qué frecuencia éstas fueron lo bastante firmes como para llevar a cabo un coito?

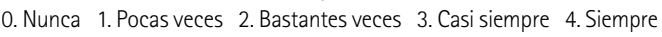

5. ¿Cuánta dificultad ha tenido, durante los últimos 30 días para conseguir una erección?

$\begin{array}{llll}0 . \text { No he tenido erecciones } & \text { 1. Mucha dificultad } & \text { 2. Alguna dificultad } & \text { 3. Poca dificultad }\end{array}$ 4. Ninguna dificultad

6. Durante los últimos 30 días, ¿Cuánta dificultad tuvo para eyacular tras haber sido estimulado sexualmente?

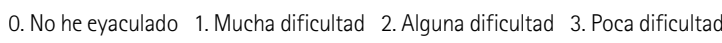
4. Ninguna dificultad

7. En los últimos 30 días, ¿En qué medida consideró problemática la cantidad de semen que usted eyaculó?

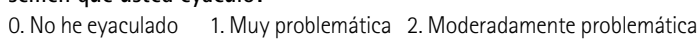

3. Poco problemática 4 . Nada problemática

8. En los últimos 30 dias, ¿En qué medida ha considerado como problemática la falta de actividad sexual?

$\begin{array}{lll}0 . \text { Muy problemática } & \text { 1. Moderadamente problemática } & 2 \text {. Poco problemática }\end{array}$

3. Muy poco problemática 4 . Nada problemática

9. En los últimos 30 días, ¿En qué medida consideró problemática su capacidad para alcanzar y mantener la erección?

0. Muy problemática $\quad$ 1. Moderadamente problemática 2. Poco problemática

3. Muy poco problemática 4 . Nada problemática

10. En los últimos 30 días, ¿En qué medida consideró su eyaculación como problemática?

0. Muy problemática $\quad$ 1. Moderadamente problemática 2 . Poco problemática

3. Muy poco problemática 4 . Nada problemática

11. En conjunto, durante los últimos 30 días, ¿Cómo de satisfecho se ha sentido con su vida sexual?

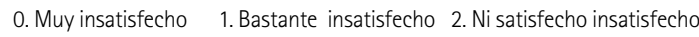

3. Bastante satisfecho 4. Muy satisfecho 